Article

\title{
(A)synchronous Communication about TV Series on Social Media: A Multi-Method Investigation of Reddit Discussions
}

\author{
Julian Unkel * and Anna Sophie Kümpel \\ Department of Media and Communication, LMU Munich, 80538 Munich, Germany; E-Mails: unkel@ifkw.Imu.de (J.U.), \\ kuempel@ifkw.Imu.de (A.S.K.) \\ * Corresponding author
}

Submitted: 20 March 2020 | Accepted: 10 June 2020 | Published: 13 August 2020

\begin{abstract}
Audiences' TV series entertainment experiences are increasingly shaped not only by the events on the 'first screen' but also by discussions on social media. While an extensive body of research has examined practices of 'second screening,' especially on Twitter, online discussions before and after the live broadcast and on other platforms have received less attention. On Reddit-one of the most important platforms for Social TV-discussions often take place in temporally structured threads that allow users to discuss an episode before (pre-premiere thread), during (live premiere thread), and after (post-premiere thread) it airs. In this project, we examine whether these spaces mainly indicate temporal preferences among users or are associated with different usage practices and motives. To do so, we conducted two case studies of the Reddit community r/gameofthrones: a survey about usage motives ( $n=417)$ and an automated content analysis of approximately 1.2 million comments left on the episode discussion threads in which we examined thread use over time, interactions between users, and discussion content. The results revealed differing usage motives and practices for the three thread types, illustrating the distinct function that these communication spaces fulfil for users.
\end{abstract}

\section{Keywords}

entertainment; multi-method; Reddit; second screen; social media; social TV; TV series; usage motives

\section{Issue}

This article is part of the issue "Computational Approaches to Media Entertainment Research" edited by Johannes Breuer (GESIS-Leibniz Institute for the Social Sciences, Germany), Tim Wulf (LMU Munich, Germany) and M. Rohangis Mohseni (TU Ilmenau, Germany).

(C) 2020 by the authors; licensee Cogitatio (Lisbon, Portugal). This article is licensed under a Creative Commons Attribution 4.0 International License (CC BY).

\section{Introduction}

In the midst of the third 'golden age of television,' we are currently experiencing a peak of quality serial television, which is characterized by complex stories and narrative structures, but also by a high degree of intertextuality and new usage and communication practices (Schlütz, 2016). In a convergent media environment, the entertainment experiences of TV series such as The Walking Dead, Doctor Who, and Game of Thrones (GoT) not only result from what happens on the 'first screen' (i.e., in the series itself), but are increasingly shaped by all the content and discussions with which users can interact on so- cial media (Raney \& Ji, 2017; Sutter, 2017). Drawing on Raney and Ji (2017, p. 428), we have reason to believe that communication about TV series on social media not only complements the TV entertainment experience, but represents "an entertainment experience, in and of itself." While funny memes or cynical comments cannot improve a bad episode, they do entertain recipients on a whole other level.

According to our review of the literature, Social TV research has mostly focused on second screening, which encompasses practices that occur "while watching television" (Gil de Zúñiga, Garcia-Perdomo, \& McGregor, 2015, p. 793), thus ignoring mediated communication that hap- 
pens prior to or after an episode airs. Considering the success of video-on-demand services (e.g., Netflix, Amazon Prime Video) and their associated viewing practices, this focus on parallel communication seems to reflect social reality less and less. Moreover, extant empirical studies seem to be centered around parallel communication on Twitter (e.g., Buschow, Schneider, \& Ueberheide, 2014; Ji \& Raney, 2015; Schirra, Sun, \& Bentley, 2014), leaving us with research gaps regarding both the temporal and platform structures of Social TV activities. In order to address these gaps, this multi-method research project: a) focuses on parallel communication (i.e., second screening) and pre- and follow-up communication; and $b$ ) investigates the usage practices and motives of these three forms of communication on Reddit, which is one of the most important platforms for Social TV (Bentley, 2017). Empirically, this research builds on two case studies of the subreddit r/gameofthrones-a discussion board for talking about GoT-and combines a user survey ( $n=417)$ with an automated content analysis of about 1.2 million user comments left in the temporally structured Reddit discussion threads (pre-premiere, live premiere, and post-premiere) about the show. This approach allows us to acquire differentiated insights into the interplay between Social TV users' motives and their actual communication practices.

\section{Social TV: Communicating about TV Series on Social Media}

In the literature, definitions of Social TV often focus on "real-time backchannel communication...during a live television broadcast" (Lim, Hwang, Kim, \& Biocca, 2015, p. 158) or "technologies that permit synchronous social interactions" (Raney \& Ji, 2017, p. 425), thus explicitly excluding asynchronous forms of communication. Drawing on Buschow et al. (2014, p. 131), we adopted a broader definition of Social TV that also includes communication practices that take place before and after a TV show airs (see Figure 1). For example, TV users might turn to Facebook groups to exchange predictions about the upcoming episodes of their favorite show (precommunication), use hashtags to follow and engage in live discussions on Twitter (parallel communication), or log in to Instagram to share memes related to key events of the most recent episode (follow-up communication).

To our knowledge, most prior studies have investigated parallel communication on Twitter, which does not adequately reflect the plethora of Social TV activities. In fact, survey studies have indicated that users seem to pre- fer asynchronous forms of communication, particularly follow-up communication (Bentley, 2017, p. 125): Asked about their latest Social TV activity, $60 \%$ of respondents indicated that they have engaged in follow-up communication. Considering the rise of streaming services, such as Netflix and Amazon Prime Video, and the practices associated with them, such as 'binge-watching' (Jenner, 2017), asynchronous Social TV activities are likely to gain even more importance. The more TV series can be watched anytime and anywhere, the more crucial understanding the uses and motives of non-parallel Social TV experiences becomes.

\subsection{Uses and Motives of Social TV}

To understand how and why users engage in (a)synchronous Social TV activities, we built on empirical research conducted in the tradition of the uses and gratifications approach (Katz, Blumler, \& Gurevitch, 1973; Rubin, 2002). Following this perspective, Social TV users can be characterized as "active, discerning, and motivated in their media use" (Quan-Haase \& Young, 2010, p. 351), resorting to specific communicative means and platforms to address diverse psychological and social needs. Social media play an increasingly important role in fulfilling these needs, as the "water cooler moments" (Lochrie \& Coulton, 2012, p. 199) associated with linear television-getting together with colleagues or acquaintances to discuss the previous night's episode-might become less frequent due to both the abundance of available shows and the mentioned changes in viewing practices (e.g., binge-watching). To put it more generally, if people want to communicate about a specific episode of a specific TV series at a specific time, social media offers targeted opportunities (Shim, Shin, \& Lim, 2017, p. 340).

Previous research on people's motivations to engage in Social TV activities has exclusively focused on parallel communication (e.g., live tweeting). Researchers have conducted both qualitative (Han \& Lee, 2014; Schirra et al., 2014) and quantitative (Gil de Zúñiga et al., 2015; Krämer, Winter, Benninghoff, \& Gallus, 2015; Shim et al., 2017) studies, thereby revealing a diverse set of affective, cognitive, personal-integrative, and social motives. For example, people engage in parallel Social TV activities to experience companionship, share their experience with other viewers, receive and/or share background information, and display their own knowledge or wit. However, we do not yet know whether these motives are more or less important in the context of asynchronous pre- and follow-up communication.

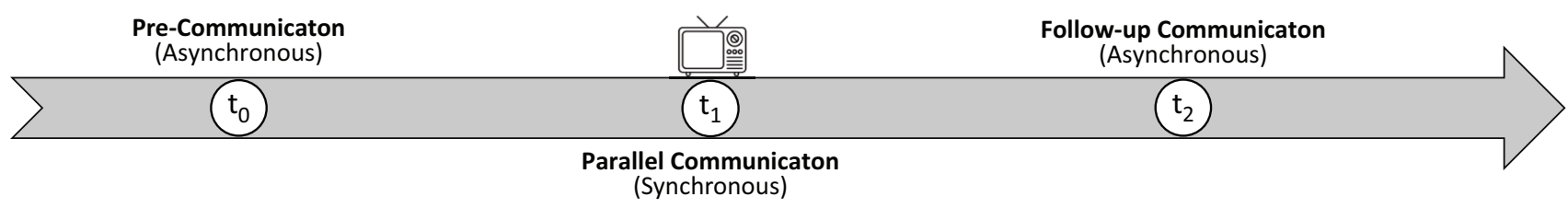

Figure 1. The Social TV experience: Forms of (a)synchronous communication about TV series on social media. 
Thus, whereas potential differences between different (a)synchronous Social TV activities have-to our knowledge-not been addressed, there are indications that channel properties and related affordances affect the uses and motives of parallel Social TV activities (Han \& Lee, 2014; Krämer et al., 2015). These studies show that using messaging apps, such as WhatsApp, is associated more with personal social needs, while using more public platforms, such as Twitter, is more strongly related to informational needs and a curiosity about other people's opinions. More generally, messaging apps seem to be used for private, in-depth conversations about TV series, whereas public social media platforms seem to be better suited for information seeking and sharing and getting a sense of a wider range of opinions. In line with these findings and the above-introduced temporal systematization of Social TV experiences, we assumed that people do not only choose specific channels/platforms in accordance with their needs but also prefer synchronous or asynchronous forms of communication for specific motives. For example, although there is mixed evidence on whether spoilers increase or decrease the enjoyment of narratives (Johnson \& Rosenbaum, 2015; Leavitt \& Christenfeld, 2013), it can be expected that some users specifically use forms of pre-communication to discuss expected developments in a show or, conversely, deliberately avoid forms of parallel communication on social media (e.g., by muting hashtags on Twitter) when they are unable to watch the live broadcast. However, it is still an open question whether meaningful differences exist in the uses and motives of different (a)synchronous Social TV activities. Thus, building on the discussed survey and interview studies (Gil de Zúñiga et al., 2015; Han \& Lee, 2014; Krämer et al., 2015; Schirra et al., 2014; Shim et al., 2017), we aim to explore diverse motivations, including the informational, social, and entertainmentrelated needs for synchronous Social TV activities established in previous research while also considering needs that might be more germane to asynchronous preand follow-up communication (e.g., creating memes, discussing spoilers).

\subsection{Reddit as a Social TV Platform}

As stated in the introduction, our research is focused on the social media platform Reddit. As the self-proclaimed "front page of the Internet," Reddit is essentially an extensive collection of thematically structured forums (known as subreddits), in which users can submit own content (e.g., texts, links, pictures, videos) in the form of posts and/or comment either directly on these original posts (top-level comments) or reply to the comments of other users, thus creating a discussion thread (Widman, 2020; also see Figure A.1 in the Supplementary File). According to recent online traffic data, Reddit is the 18th most popular website in the world, with most of its desktop traffic originating from the US (49.9\%), the UK (7.9\%), and Canada (7.5\%; Alexa, 2020; SimilarWeb, 2020). In addi- tion to its popularity among (English-speaking) Internet users, Reddit has become an attractive data source (Amaya, Bach, Keusch, \& Kreuter, 2019) because it is: a) mostly public and can be browsed by unregistered users; b) allows the identification and study of special or otherwise hard-to-recruit and -observe populations; and c) is perceived as suitable in investigations of users' "true beliefs" (Amaya et al., 2019, p. 2) due to its largely anonymous environment.

Even more pertinent to this research is the fact that Reddit is highly relevant to Social TV (Bentley, 2017; Cassis, 2018). In Bentley's survey (2017, p. 125), $47 \%$ of respondents (19- to 69-year-old US Americans recruited via Amazon Mechanical Turk) said that they had turned to Reddit in the past month to find additional information about a TV show, actor, or character, making it the most important social media platform for such activities. Moreover, Reddit is ideal for simultaneously investigating asynchronous (pre-communication, follow-up communication) and synchronous (parallel communication) forms of communication. To facilitate separated discussions before (pre-premiere thread), during (live premiere thread), and after (post-premiere thread) an episode airs, many TV subreddits (e.g., those dedicated to GoT, The Walking Dead, and Doctor Who) offer temporally structured threads that mirror the conceptual tripartition of Social TV activities (see Figure 1). This tripartition of threads allows us to get a sense of usage motives and practices while keeping possible influences of platform affordances or user characteristics more or less constant.

Building on the prior discussion of the uses and motives of Social TV and given the scarcity of research on asynchronous Social TV activities on platforms other than Twitter, our project was guided by two overarching research questions:

RQ1: Usage motives: What motivates users to engage in (a)synchronous communication about TV series on Reddit?

RQ2: Usage practices: What characterizes (a)synchronous communication about TV series on Reddit?

\section{Method}

To address these research questions, we conducted a multi-method case study of r/gameofthrones, which is the largest GoT subreddit and has been among the most-frequented TV-centric subreddits, with about 11.5 million unique users per month in 2018 (Cassis, 2018). Episode discussion threads on r/gameofthrones have followed the aforementioned tripartition into prepremiere, live premiere, and post-premiere discussion threads since the middle of Season 4. All three thread types are 'official' discussion threads created by the moderators or select subreddit users, meaning that every episode has a dedicated pre-premiere, live premiere, and post-premiere thread. For this case study, we drew from 
two data sources that will be described in more detail below: the content and meta-textual features of all comments posted in the episode discussion threads for Seasons 5-8, and a survey of subreddit users conducted during the airing of the eighth and final season. The data and reproducible $R$ analysis scripts can be obtained from this study's Open Science Framework (OSF) repository: https://osf.io/7v49t/.

\subsection{Comments on Subreddit Discussion Threads}

There are two main ways to access organic (i.e., usergenerated) Reddit data: through the official Reddit application programming interface (API) and the Pushshift Reddit dataset (Amaya et al., 2019). While both generally provide the same data, we opted for the latter, as it provides a much easier and more flexible way of querying Reddit data (e.g., higher rate limits). The Pushshift Reddit dataset (Baumgartner, Zannettou, Keegan, Squire, \& Blackburn, 2020) is a privately maintained dataset that ingests Reddit data in real time and contains all posts and comments made on the platform since June 2005. Data are split into two files (one for original posts and the other for comments) and may be accessed in a variety of ways, including monthly dumps downloadable directly from the Pushshift website and an open API. For this project, we wrote a simple $R$ function that, for a given list of Reddit posts (identified via IDs that are also part of each post's URL), queries the Pushshift API and returns all comments posted in those threads, including associated meta-textual information (e.g., creation time, parent comment ID, comment score). Data are returned in JavaScript Object Notation format, which can be easily converted to various tabular data frame formats for further analysis. All API querying and data handling steps were conducted using various functions from the "Tidyverse" meta package (Wickham et al., 2019).

We queried the Pushshift API for all comments posted in the episode discussion threads for seasons 5-8, resulting in 1,252,971 individual comments. All queries were performed in September 2019, about three months after the broadcast of the final GoT episode. All comments either deleted by the original author or removed by a moderator between its original post date and the query date as well as all comments by discussion moderation bots were removed from the data, leading to a final sample of $1,203,666$ comments from 243,997 unique users.

\subsection{Survey of Subreddit Users}

A survey of 417 users of r/gameofthrones was conducted from April 11, 2019-three days before the US premiere of the first episode of Season 8-to April 22, 2019. Prospective participants were recruited by posts and sponsored links on Reddit. Participation was completely voluntary, anonymous, and unincentivized. The majority of participants (58\%) self-identified as male
(41\% female; $1 \%$ non-binary) and were rather young $(M=28.30$ years, $S D=8.75)$. Further sample demographics included education (61\% university degree) and nationality (58\% US citizens).

A list of 25 subreddit usage motives (see Table 1) was compiled based on previous Social TV studies (Gil de Zúñiga et al., 2015; Han \& Lee, 2014; Krämer et al., 2015; Puschmann, 2017; Shim et al., 2017). Participants were first asked to rate the importance of each of these motives to their personal subreddit use on a Likerttype scale from 1 (strongly disagree) to 7 (strongly agree). Afterwards, participants were presented the same list again and asked to select which, if any, of the three thread types (pre-premiere, live premiere, postpremiere) was best suited to fulfilling each motive. The full survey questionnaire and an overview of all motive ratings are available on the study's OSF repository.

\section{Results}

\subsection{Usage Motives of Discussion Threads}

In order to identify distinct motive factors, we conducted an exploratory maximum likelihood factor analysis with oblique rotation on the 25 surveyed usage motives using the $R$ package "psych" (Revelle, 2019). We retained five factors based on a preceding parallel analysis (see Table 1). The most important motive factor for r/gameofthrones subreddit users was 'Knowledge' ( $M=5.60, S D=1.19$, 7-point agreement scale), which spoke to users' need to obtain background lore and expand their knowledge. This factor was followed by 'Humor' $(M=5.09, S D=1.79)$, which represented the need for entertainment through memes and humorous comments. Virtual 'Company' $(M=3.14, S D=1.72)-$ the feeling of a shared viewing experience and the social gratifications connected with it-played a rather minor role, as did the needs to actively contribute one's own content, theories, or comments ('Share,' $M=2.34$, $S D=1.42$ ) and to share/receive spoilers ('Spoilers,' $M=2.02, S D=1.35$ ).

However, thread type suitability ratings indicated that the temporally structured discussion threads did indeed appear to be differently able to fulfil usage motives (see Figure 2). Although the post-premiere thread seemed to be the 'all-rounder' and suited to fulfilling all five overarching motive factors, those motives related to the need for company could be best satisfied by parallel communication in the live-premiere thread. The prepremiere thread, on the other hand, was mostly relevant to refreshing one's knowledge or receiving background information but seemed to be of limited relevance to the other motive factors. Crucially, for each motive, a substantial share of participants did not specify a most-adequate thread type (ranging from $14.9 \%$ for Motive 14 ["know whether I have missed something important"] to $46.3 \%$ for Motive 08 ["earn some Reddit karma/silver/gold"]). This may be attributable to partic- 


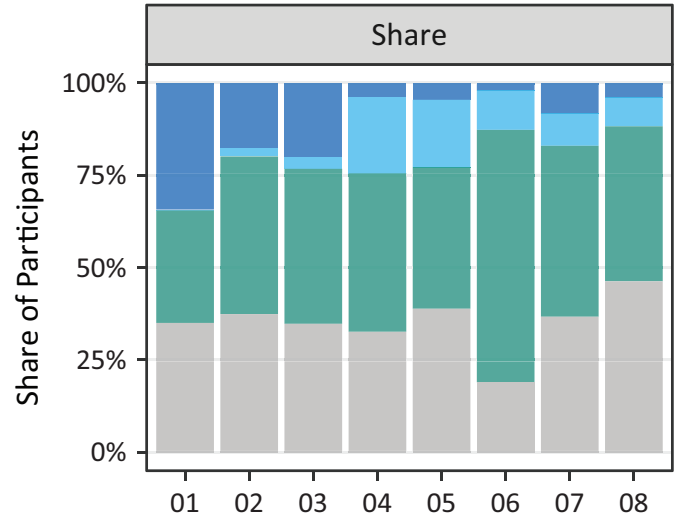

Thread Type
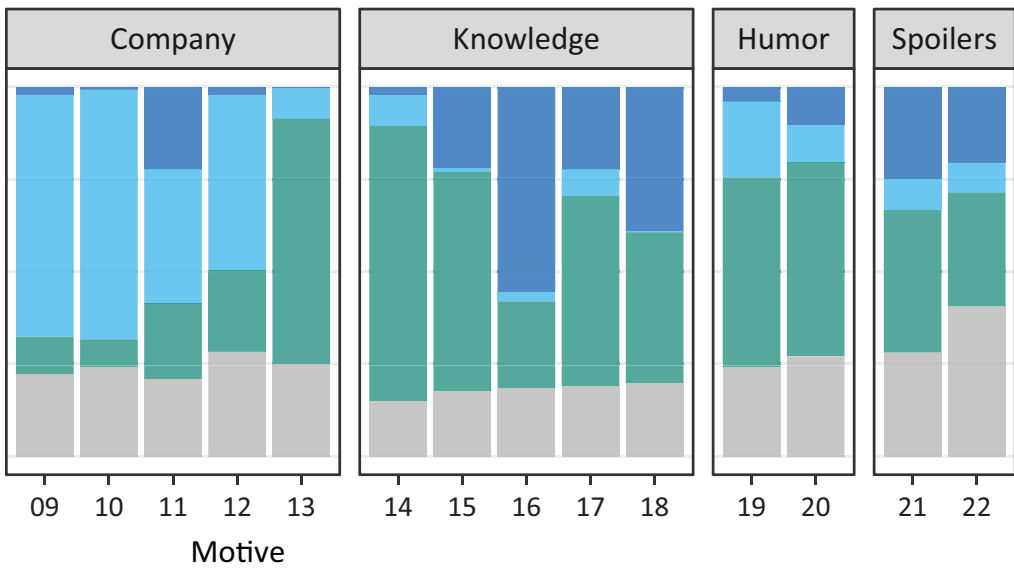

$\begin{array}{llll}19 & 20 & 21 & 22\end{array}$

Figure 2. Thread most suitable to fulfilling usage motives. Notes: $n=417$. For motive item wordings see Table 1.

ipants perceiving the respective motives as less important and/or to users' perception of multiple thread types as equally suitable to fulfilling these motives.

\subsection{Usage Practices of Discussion Threads}

To investigate whether the temporally structured discussion spaces were also characterized by differing usage practices, we turned to the content and meta-textual information of the comments left in those threads. In purely quantitative terms, the three thread types were used very differently (see Figure 3 ). While the popularity of all thread types increased over time, by far the most comments were found in the post-premiere threads, with all Season 8 episodes generating more than 40,000 comments (and peaking at around 100,000 comments for the controversial episode "The Long Night"). This was followed by synchronous live commenting on the episodes. The pre-premiere threads rarely exceeded
5,000 comments. Of course, the temporal structuring of the threads predefined when comments were made in relation to the episode's airing, with the pre-premiere thread starting 48 hours before the US premiere of an episode and the live and post-premiere threads appearing shortly before the start and the end of each episode. Thus, despite the possibility of asynchronous communication on the episodes, thread usage was strongly influenced by linear program planning. All three threads received the most attention in a window of only a few hours immediately before and after the US premiere of the respective episode (see Figure 4). Even in the postpremiere thread, the vast majority of comments were made within three to four hours after the episode aired, and almost no new comments appeared after about a day.

Looking closer at the content of the comments, we found that the use of the live premiere threads in particular differed from the asynchronous thread types. Not

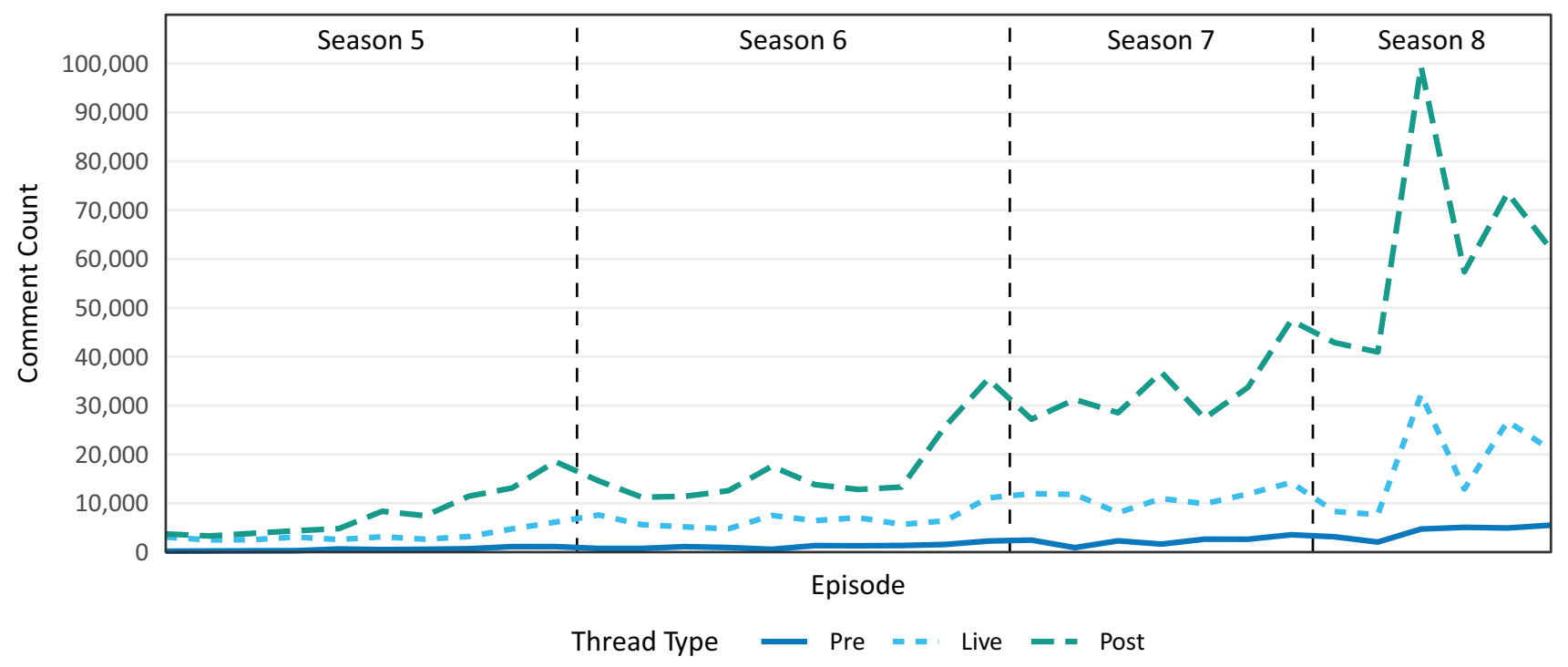

Figure 3. Comment counts per episode and thread type. Note: $n=1,203,666$. 
Table 1. Factor loadings of thread usage motives.

\begin{tabular}{|c|c|c|c|c|c|}
\hline I use the discussion threads to... & $\begin{array}{c}\text { I } \\
\text { Share }\end{array}$ & $\begin{array}{c}\text { II } \\
\text { Company }\end{array}$ & $\begin{array}{c}\text { III } \\
\text { Knowledge }\end{array}$ & $\begin{array}{l}\text { IV } \\
\text { Humor }\end{array}$ & $\begin{array}{c}\text { V } \\
\text { Spoilers }\end{array}$ \\
\hline 01: share background lore & 0.885 & -0.155 & 0.103 & -0.046 & -0.069 \\
\hline $\begin{array}{l}\text { 02: share my own theories about developments } \\
\text { in the series }\end{array}$ & 0.853 & -0.103 & 0.079 & -0.101 & -0.051 \\
\hline 03: show my knowledge about the series & 0.808 & 0.086 & -0.011 & -0.085 & -0.034 \\
\hline 04: post humorous comments & 0.793 & -0.051 & 0.004 & 0.144 & -0.076 \\
\hline 05: show my wit and humor & 0.765 & 0.047 & -0.050 & 0.062 & 0.044 \\
\hline 06: discuss or argue about events in the episode & 0.722 & 0.142 & 0.006 & -0.131 & -0.146 \\
\hline 07: post or share memes & 0.519 & -0.073 & -0.083 & 0.320 & 0.143 \\
\hline 08: earn some Reddit karma/silver/gold & 0.423 & -0.028 & -0.048 & 0.036 & 0.222 \\
\hline 09: feel like watching together with other people & -0.126 & 0.957 & -0.083 & 0.003 & -0.033 \\
\hline 10: not be alone while watching & -0.148 & 0.846 & -0.153 & -0.002 & 0.084 \\
\hline 11: share excitement or suspense & 0.271 & 0.626 & 0.036 & -0.044 & -0.112 \\
\hline 12: interact with other people & 0.395 & 0.586 & -0.055 & -0.115 & 0.031 \\
\hline 13: deal or cope with tragic events in the series & 0.011 & 0.572 & 0.082 & 0.025 & -0.080 \\
\hline $\begin{array}{l}\text { 14: know whether I have missed something } \\
\text { important }\end{array}$ & -0.020 & 0.092 & 0.510 & 0.052 & 0.028 \\
\hline 15: read theories about developments in the series & 0.011 & -0.011 & 0.406 & 0.012 & 0.011 \\
\hline $\begin{array}{l}\text { 16: refresh my knowledge about past developments } \\
\text { in the series }\end{array}$ & 0.005 & -0.026 & 0.742 & -0.081 & 0.058 \\
\hline $\begin{array}{l}\text { 17: receive specific information about characters, } \\
\text { locations, music, etc. }\end{array}$ & 0.050 & -0.069 & 0.736 & 0.025 & 0.064 \\
\hline 18: read background lore & 0.015 & -0.080 & 0.831 & -0.038 & -0.035 \\
\hline 19: read humorous comments & 0.024 & 0.038 & 0.030 & 0.755 & -0.120 \\
\hline 20: read or view memes & -0.082 & -0.005 & -0.027 & 0.872 & -0.030 \\
\hline 21: read spoilers & -0.172 & -0.044 & 0.145 & -0.060 & 0.695 \\
\hline 22: share spoilers & 0.263 & -0.094 & -0.022 & -0.048 & 0.552 \\
\hline 23: bypass boring moments & 0.000 & 0.157 & -0.053 & -0.011 & 0.250 \\
\hline 24: get to know about other people's opinions & -0.029 & 0.297 & 0.193 & 0.091 & 0.003 \\
\hline 25: get myself in the mood for watching & 0.029 & 0.311 & 0.149 & 0.039 & 0.091 \\
\hline$\%$ Variance & 0.19 & 0.12 & 0.09 & 0.06 & 0.04 \\
\hline$M(S D)$ & $2.34(1.42)$ & $3.14(1.72)$ & $5.60(1.19)$ & $5.09(1.79)$ & $2.02(1.35)$ \\
\hline$\omega_{\mathrm{h}}$ & 0.90 & 0.84 & 0.79 & 0.78 & 0.58 \\
\hline
\end{tabular}

Notes: Maximum likelihood factor analysis with oblique rotation (Promax) for all 25 motive items. Scale: 1 (strongly disagree) to 7 (strongly agree). $n=417$, Kaiser-Meyer-Olkin measure (KMO) $=0.87$ (all KMO values for individual items $\geq 0.68$ ), Bartlett's $\mathrm{K}^{2}(24)=808.34, p<0.001$; parallel analysis suggested five factors. All factor loadings $<|0.4|$ grayed out.

only were the comments in the live premiere threads on average much shorter $(M=76$ characters, $S D=118)$ than in the pre- $(M=142, S D=231)$ and post-premiere threads $(M=126, S D=189)$, there was less interaction between users, as indicated by the fact that more than half $(52 \%)$ of all comments in the live premiere threads were top-level comments-comments that replied to the original post and thus did not directly engage with comments from other users. In contrast, top-level comments only accounted for about one-fifth to a quarter of all comments in the pre- $(24 \%)$ and post-premiere threads (21\%).

To further investigate the content of the comments, we focused on keywords, that is, words that were distinctive in each thread type. Using the $R$ package "quanteda" (Benoit et al., 2018), we first preprocessed all comments by removing URLs, symbols (including emo- jis), stopwords based on the Snowball stopword list, and further uninformative terms that were identified during preliminary data analysis (e.g., all-text emojis such as " $x D$ " and remaining parts of HTML entities like '\&amp;' and '\&gt;'). We then calculated log-likelihood keyness scores for each thread type, using the other two thread types as reference groups. The identified keywords (see Figure 5) offered insights into the differences in discussion content. Comments in the pre-premiere thread seem to focus on predictions and discussions of (leaked) episode plot points and developments (e.g., "predict(ion/s)," "theory," "kills," "dies," "leaked," and "spoilers") and on the framing of the individual reception situation (e.g., "watch," "see," "premiere," and "hbo;" see also Puschmann, 2017). Live premiere threads were characterized by expletives and exclamations of surprise (e.g., "omg," "oh," and various four-letter words). Finally, 

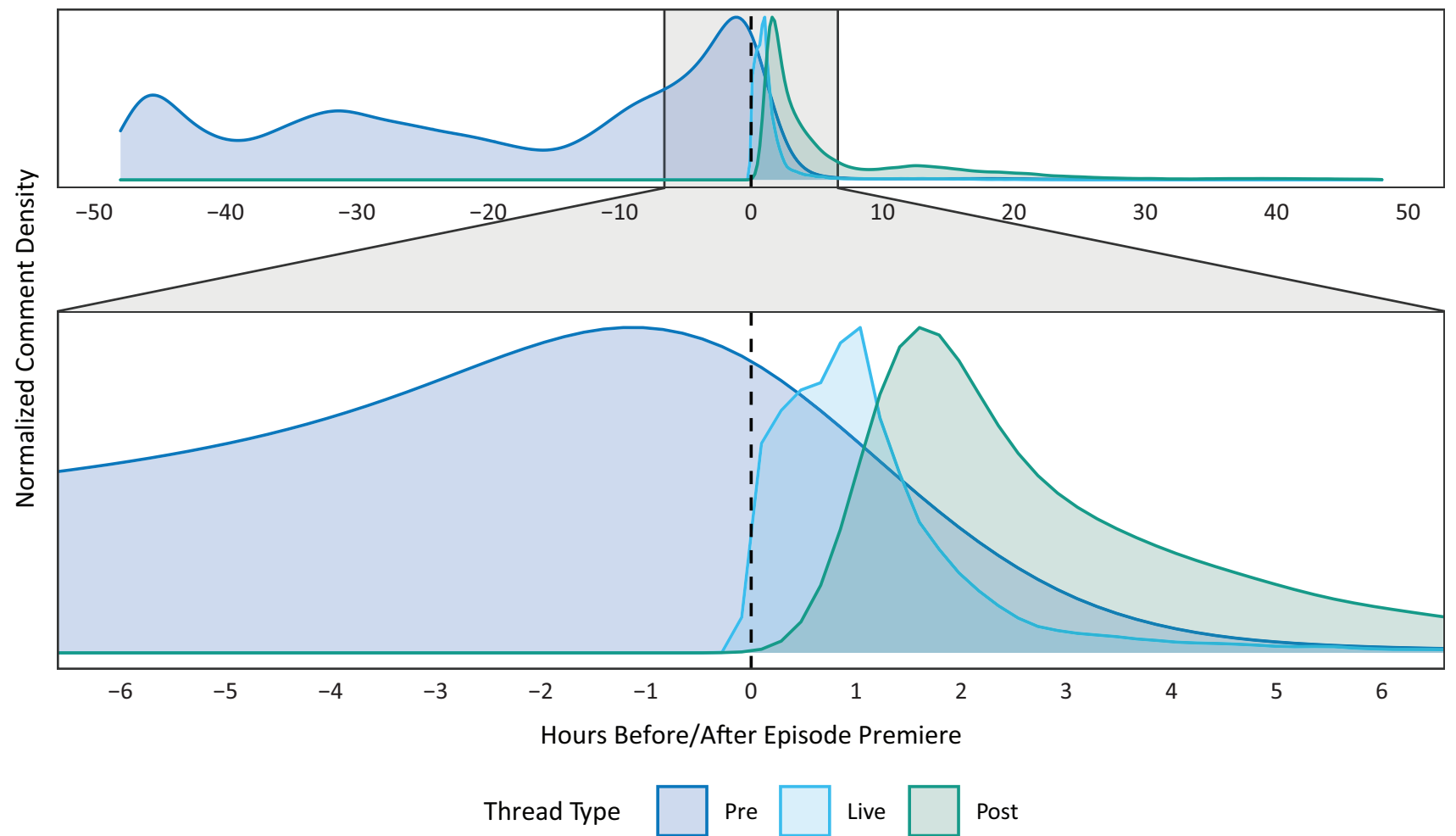

Figure 4. Comment density in relation to US episode premiere. Note: $n=1,203,666$.

the post-premiere threads contained more in-depth discussions and evaluations of recent series events (e.g., "loved," "felt," "thought," and "scene"); crucially, other Reddit users and their opinions were explicitly addressed and consulted (e.g., "anyone" and "else").

\section{Discussion and Conclusion}

The present study attempted to explore which usage motives and practices are characteristic in different forms of asynchronous and synchronous communication about
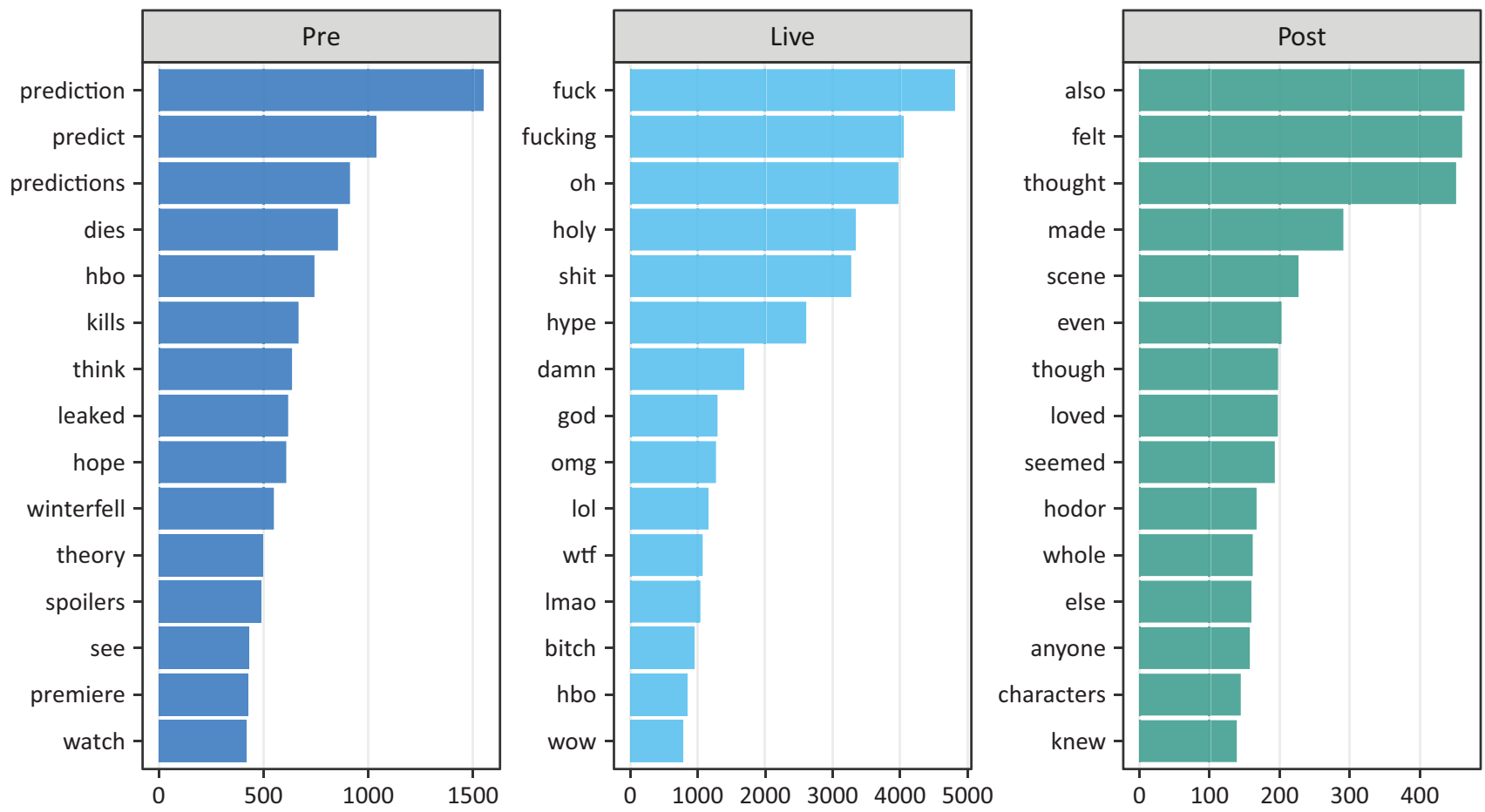

G2

Figure 5. Keywords with highest likelihood-ratio G2 score per thread type. 
TV series on social media. Building on prior research, we simultaneously examined the uses and motives of precommunication, live communication, and follow-up communication on Reddit, a highly important yet understudied platform in the context of Social TV activities. Our multi-method research project combined an automated content analysis of more than 1.2 million user comments from the subreddit r/gameofthrones-an online community centered on discussions of the HBO series GoTwith a survey of users active in this community $(n=417)$.

While prior research already found that messaging apps and more public social media platforms are used differently and for different reasons in the context of Social TV (Han \& Lee, 2014; Krämer et al., 2015), our project has shown that discussion spaces dedicated to asynchronous and synchronous communication about TV series are also frequented and valued for different reasons. Focusing on our case study of discussions about GoT, we found that refreshing and extending one's knowledge of the show (factor 'Knowledge') and viewing memes and humorous comments (factor 'Humor') were overall the most important for users of the r/gameofthrones subreddit. However, the temporally structured threads (pre-, live-, and post-premiere thread) were perceived to be more or less suited to fulfilling specific motives. According to our participants, the need for feeling like they were watching with others and not being alone (factor 'Company') was best satisfied by parallel communication in the live premiere thread, while almost all motives related to sharing one's own information or theories (factor 'Share') were perceived as best fulfilled in the post-premiere thread. Thus, not all Social TV activities are created equal, and users seem to engage in these activities with the goal of addressing specific needs and desires. This also became apparent when we considered the broader information environment on Reddit. While r/gameofthrones is by far the most frequented and broadest GoT-related subreddit, there are also specialized subreddits that are, for example, exclusively focused on humorous content and memes (e.g., r/aSongOfMemesAndRage).

To get a more detailed picture, we complemented the survey with a large-scale automated content analysis of actual usage practices (i.e., commenting behavior) in the three thread types. This analysis has shown that: (1) follow-up communication in the postpremiere threads was most popular among users of r/gameofthrones; (2) parallel communication in the live premiere threads was less extensive and less interactive than pre-communication and follow-up communication; and (3) the focus and content of discussions varied in the three threads as well. Looking at the most important terms per thread type, we found that the post-premiere threads seemed to be characterized by users sharing background information and wanting to discuss theories, as other users were addressed explicitly and specific developments were debated; this mirrored our survey results. The live premiere threads, on the other hand, were used to express one's surprise and emotions, while discussions in the pre-premiere threads were indicative of talking about one's ability to watch the current episode and for exchanging predictions.

Taken together, our multi-method approach allowed us to gain differentiated insights into (a)synchronous Social TV activities and has shown the potential of combining survey and content data. This potential is best illustrated by an example from our data: if we had relied solely on the survey data, we might have suspected that the importance of the live premiere thread to the motive factor 'Company' (i.e., feeling like watching together, sharing one's feelings with others) would have led users to engage in lively exchanges and more interactions with others. Instead, the thread analysis indicated that comments in the live premiere threads were the shortest, and the level of direct interactions was, by far, the lowest. This finding implies that the need for company might already be stimulated by the mere feeling of being in the same (online) space as other viewers-comparable to the collective cinema experience of sitting and watching in silence, united simply by joint attention to the screen (Hanich, 2018). Direct interactions with other users happened more often-and were actively sought out-in the asynchronous discussion threads. On the other hand, the survey responses provided us with deeper insights into the users' motives in addition to the discussion content, especially as the automated content analysis faced some challenges and limitations. Specifically, at least with data-driven, unsupervised text analysis methods, we were not able to extract discussion patterns in the actual Reddit comments that showed substantial overlap with the surveyed usage motives. In future studies, more theory-based analysis procedures such as dictionaries or adaptations of topic modeling procedures that allow for the prior specification of keywords (e.g., Eshima, Imai, \& Sasaki, 2020) might provide viable alternatives in the more meaningful combination of user-centric survey results and content-centric automated text analysis.

Although the foci on a single platform, a single community, and a single TV series reduces the generalizability of our findings, this case study approach allowed us to gain insights into distinct Social TV activities while reducing the influence of confounding variables introduced by platform affordances (e.g., number of characters allowed in comments), discussion dynamics (e.g., users' mode of interacting on Instagram versus on Reddit), or audience characteristics. Future research should build on our findings by investigating pre-communication, parallel communication, and follow-up communication on different social media and with a focus on different TV series. For example, it might be expected that viewers of TV series that are less 'story-heavy' than GoT have a diminished need for background knowledge and theories, but are even more interested in humorous interactions or memes. However, due to the fact that the show's content "serves as the source material for content shared on the second screen" (Raney \& Ji, 2017, p. 429), it will prob- 
ably never be possible to completely separate Social TV activities from the TV series they relate to.

With the success of streaming services and viewing behaviors that are less and less tied to single episodes, the necessity to investigate asynchronous Social TV activities will certainly increase. For example, as the episodes of most shows of subscription-based video-on-demand services, such as Netflix and Amazon Prime Video, are released en bloc, parallel communication becomes less important because there is no actual 'live' moment anymore, not even for users in the same time zone. Popular discussions indicate that tweeting behavior seems to be particularly challenged by this all-at-once release strategy (see Weller, 2016): indeed, when, if at all, is it acceptable to post about a new Netflix show on Twitter-and what is one allowed to give away? Considering these debates in the context of our findings, we would particularly encourage a closer look at forms of follow-up communication, as the post-premiere threads consistently emerged as the most frequented ones. Moreover, it is likely that the engagement in Social TV discussions also alters the experience of first screen content (Krämer et al., 2015; Raney \& Ji, 2017). As such, differences in Social TV usage motives and practices may lead to differential effects on users' entertainment experiences. From a methodological standpoint, it is interesting to consider that these entertainment experiences are likely to be reflected in the content of Social TV discussions, as indicated by expressions of suspense, surprise, or shock in the live episode discussion threads and in-depth discussions of affective responses in the post-premiere threads. Being able to identify the different entertainment experiences of TV series/episodes (e.g., hedonic and eudaimonic, see Oliver \& Raney, 2011; Vorderer, 2011) by observing discussions on social media would benefit media entertainment research by reducing the need to rely on retrospective self-reports. As a first step in this direction, dictionary-based methods may provide a scalable procedure to investigate hedonic and eudaimonic entertainment experiences based on user comments.

Despite the inherent limitations of our project, this research presents exploratory first steps in understanding the different temporal, motivational, and behavioral facets of Social TV experiences. By recognizing that communication about TV series in social media goes beyond synchronous interactions, media entertainment research will be in a better position to explore the gratifications of reading, learning, and talking about one's favorite TV show. After all, TV has always been a social experience and will continue to be so in online environments.

\section{Acknowledgments}

The authors wish to thank the four anonymous reviewers and the thematic issue editors for their valuable comments.

\section{Conflict of Interests}

The authors declare no conflict of interests.

\section{Supplementary Material}

Supplementary material for this article is available online in the format provided by the authors (unedited). The replication code and data are available at https://osf.io/7v49t/.

\section{References}

Alexa. (2020). The top 500 sites on the web. Alexa. Retrieved from https://www.alexa.com/topsites

Amaya, A., Bach, R., Keusch, F., \& Kreuter, F. (2019). New data sources in social science research: Things to know before working with Reddit data. Social Science Computer Review. Advance online publication. https://doi.org/10.1177/0894439319893305

Baumgartner, J., Zannettou, S., Keegan, B., Squire, M., \& Blackburn, J. (2020). The pushshift Reddit Dataset. Proceedings of the International AAAl Conference on Web and Social Media, 14(1), 830-839. Retrieved from https://www.aaai.org/ojs/index.php/ ICWSM/article/view/7347

Benoit, K., Watanabe, K., Wang, H., Nulty, P., Obeng, A., Müller, S., \& Matsuo, A. (2018). quanteda: An R package for the quantitative analysis of textual data. Journal of Open Source Software, 3(30), 774. https://doi. org/10.21105/joss.00774

Bentley, F. R. (2017). Understanding secondary content practices for television viewing. In Proceedings of the 2017 ACM International Conference on Interactive Experiences for TV and Online Video (pp. 123-128). New York, NY: Association for Computing Machinery. https://doi.org/10.1145/3077548.3077554

Buschow, C., Schneider, B., \& Ueberheide, S. (2014). Tweeting television: Exploring communication activities on Twitter while watching TV. Communications, 39(2), 129-149. https://doi.org/10.1515/commun2014-0009

Cassis, C. (2018). Discuss and discover TV with Reddit. Upvoted. Retrieved from https://redditblog.com/2018/ 08/10/discuss-and-discover-tv-with-reddit

Eshima, S., Imai, K., \& Sasaki, T. (2020). Keyword assisted topic models. Unpublished manuscript. Retrieved from http://arxiv.org/abs/2004.05964

Gil de Zúñiga, H., Garcia-Perdomo, V., \& McGregor, S. C. (2015). What is second screening? Exploring motivations of second screen use and its effect on online political participation. Journal of Communication, 65(5), 793-815. https://doi.org/10.1111/jcom.12174

Han, E., \& Lee, S.-W. (2014). Motivations for the complementary use of text-based media during linear TV viewing: An exploratory study. Computers in Human Behavior, 32, 235-243. https://doi.org/ 10.1016/j.chb.2013.12.015 
Hanich, J. (2018). Audience effect: On the collective cinema experience. Edinburgh: Edinburgh University Press.

Jenner, M. (2017). Binge-watching: Video-on-demand, quality TV and mainstreaming fandom. International Journal of Cultural Studies, 20(3), 304-320. https:// doi.org/10.1177/1367877915606485

Ji, Q., \& Raney, A. A. (2015). Morally judging entertainment: A case study of live tweeting during Downton Abbey. Media Psychology, 18(2), 221-242. https:// doi.org/10.1080/15213269.2014.956939

Johnson, B. K., \& Rosenbaum, J. E. (2015). Spoiler alert: Consequences of narrative spoilers for dimensions of enjoyment, appreciation, and transportation. Communication Research, 42(8), 1068-1088. https://doi. org/10.1177/0093650214564051

Katz, E., Blumler, J. G., \& Gurevitch, M. (1973). Uses and gratifications research. The Public Opinion Quarterly, 37(4), 509-523.

Krämer, N. C., Winter, S., Benninghoff, B., \& Gallus, C. (2015). How "social" is Social TV? The influence of social motives and expected outcomes on the usage of Social TV applications. Computers in Human Behavior, 51, 255-262. https://doi.org/10.1016/j.chb. 2015.05.005

Leavitt, J. D., \& Christenfeld, N. J. S. (2013). The fluency of spoilers: Why giving away endings improves stories. Scientific Study of Literature, 3(1), 93-104. https:// doi.org/10.1075/ssol.3.1.09lea

Lim, J. S., Hwang, Y., Kim, S., \& Biocca, F. A. (2015). How social media engagement leads to sports channel loyalty: Mediating roles of social presence and channel commitment. Computers in Human Behavior, 46, 158-167. https://doi.org/10.1016/j.chb.2015.01. 013

Lochrie, M., \& Coulton, P. (2012). Sharing the viewing experience through second screens. In Proceedings of the 10th European Conference on Interactive TV and Video (pp. 199-202). New York, NY: Association for Computing Machinery. https://doi.org/10.1145/ 2325616.2325655

Oliver, M. B., \& Raney, A. A. (2011). Entertainment as pleasurable and meaningful: Identifying hedonic and eudaimonic motivations for entertainment consumption. Journal of Communication, 61(5), 984-1004. https://doi.org/10.1111/j.1460-2466.2011.01585.x

Puschmann, C. (2017). Beitragstypen der öffentlichen rezeptionsbegleitenden Kommunikation auf Twitter bei fiktionalen TV-Inhalten [Types of tweets in the public, synchronous communication about fictional TV content on Twitter]. In U. Göttlich, L. Heinz, \& M. R. Herbers (Eds.), Ko-Orientierung in der Medienrezeption: Praktiken der Second-Screen-Nutzung [Coorientation in media use: Practices of second screen use] (pp. 195-218). Cham: Springer.

Quan-Haase, A., \& Young, A. L. (2010). Uses and gratifications of social media: A comparison of Facebook and instant messaging. Bulletin of Science, Technology
\& Society, 30(5), 350-361. https://doi.org/10.1177/ 0270467610380009

Raney, A. A., \& Ji, Q. (2017). Entertaining each other? Modeling the socially shared television viewing experience. Human Communication Research, 43(4), 424-435. https://doi.org/10.1111/hcre.12121

Revelle, W. psych: Procedures for psychological, psychometric, and personality research (Version 1.9.12) [Computer software]. (2019). Evanston, IL: Northwestern University. Retrieved from https://CRAN.Rproject.org/package=psych

Rubin, A. M. (2002). The uses-and-gratifications perspective of media effects. In J. Bryant \& D. Zillmann (Eds.), Media effects: Advances in theory and research (2nd ed.) (pp. 525-548). Mahwah, NJ: Lawrence Erlbaum Associates.

Schirra, S., Sun, H., \& Bentley, F. (2014). Together alone: Motivations for live-tweeting a television series. In CHI '14: Proceedings of the SIGCHI Conference on Human Factors in Computing Systems (pp. 2441-2450). New York, NY: Association for Computing Machinery. https://doi.org/10.1145/2556288.2557070

Schlütz, D. (2016). Contemporary quality TV: The entertainment experience of complex serial narratives. Annals of the International Communication Association, 40(1), 95-124. https://doi.org/10.1080/ 23808985.2015.11735257

Shim, H., Shin, E., \& Lim, S. (2017). What makes us twoscreen users? The effects of two-screen viewing motivation and psychological traits on social interactions. Computers in Human Behavior, 75, 339-346. https:// doi.org/10.1016/j.chb.2017.05.019

SimilarWeb. (2020). Reddit.com. SimilarWeb. Retrieved from http://similarweb.com/website/reddit.com

Sutter, T. (2017). Kommunikation über Fernsehen im Internet. Social TV als Anschlusskommunikation [Communication about TV on the Internet. Social TV as follow-up communication]. In U. Göttlich, L. Heinz, \& M. R. Herbers (Eds.), Ko-Orientierung in der Medienrezeption: Praktiken der Second-Screen-Nutzung [Coorientation in media use: Practices of second screen use] (pp. 29-46). Cham: Springer.

Vorderer, P. (2011). What's next? Remarks on the current vitalization of entertainment theory. Journal of Media Psychology, 23(1), 60-63. https://doi.org/ 10.1027/1864-1105/a000034

Weller, C. (2016, July 5). The complicated ethics of tweeting about your favorite Netflix show. Business Insider. Retrieved from https://www.businessinsider. com/ethics-of-tweeting-about-netflix-show-2016-7

Wickham, H., Averick, M., Bryan, J., Chang, W., McGowan, L., François, R., . . . Yutani, H. (2019). Welcome to the Tidyverse. Journal of Open Source Software, 4(43), 1686. https://doi.org/10.21105/ joss.01686

Widman, J. (2020, July 1). What is Reddit? Digital Trends. Retrieved from https://www.digitaltrends. com/web/what-is-reddit 


\section{About the Authors}

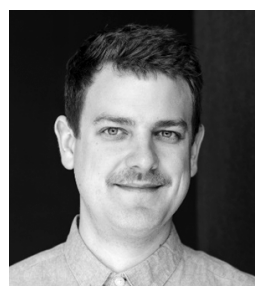

Julian Unkel (Dr. rer. Soc., LMU Munich) is a Postdoctoral Researcher at the Department of Media and Communication at LMU Munich. His research interests focus on online media selection and effects, media entertainment, and communication research methods. As a member of the LMU Open Science Center, he seeks to promote and to foster open science practices in communication research. More information: http://julianunkel.com

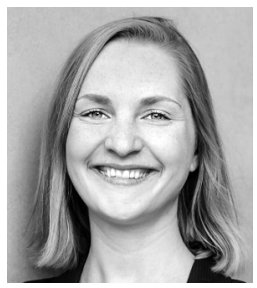

Anna Sophie Kümpel (Dr. rer. soc., LMU Munich) is a Postdoctoral Researcher at the Department of Media and Communication at LMU Munich. Her research interests are focused on media effects, particularly in the context of social media, (incidental exposure to) online news, and media entertainment. Her research has been published in Journal of Communication, Journal of Media Psychology, and Social Media + Society, among others. More information: http://anna-kuempel.de 